\title{
Performances Evaluation of Inverse Kinematic Models of a Compact Bionic Handling Assistant
}

\author{
Inderjeet Singh $^{1}$, Othman Lakhal ${ }^{1}$, Yacine Amara ${ }^{2}$, Vincent Coelen ${ }^{1}$, Pushparaj Mani Pathak ${ }^{3}$ and Rochdi Merzouki ${ }^{1}$
}

\begin{abstract}
Inverse Kinematic Model (IKM) is very crucial for real-time control of a robot for any application. Computing the IKM of continuum manipulators is a challenging task. Two types of methods exists; quantitative methods describing a model-based approach; and qualitative methods, based on learning approach. As quantitative methods are based on mathematical expression, they are more flexible for extension (increase in number of sections or collaboration between more than one manipulator). In this paper, two quantitative approaches based on Newton Raphson iterative method and Damped Least Square method, are proposed for Compact Bionic Handling Assistant (CBHA) manipulator to solve inverse kinematics directly using Forward Kinematic Model (FKM). Experimental validation is done for these methods as well as they are compared with the existing approaches named Hybrid approach and Neural Network based learning approach.
\end{abstract}

\section{INTRODUCTION}

In robotics, bionic continuum manipulators is a rapidly growing field. Bionic manipulators are inspired from many similar biological entities like octopus [1], elephant trunk [2] etc. Modeling of continuum manipulators is complex owing to their non-linear nature, high degree of freedom, and soft material. The soft material accounts for the flexibility in the structure which allows the manipulator to work even in very congested environments [3].

Quantitative models have been studied extensively. A threedimensional kinematic model is represented by [4], for multi-section continuum arms, with the help of novel shape function based approach. An intuitive kinematic model is developed for a continuum manipulator by using DenavitHartenberg method and differential geometry [5]. For this approach, the curvature of backbone is assumed circular. The same approach is also used in [6] to model the kinematics of a manipulator which is based on tendon. Forward kinematic model (FKM) for Compact Bionic Handling Assistant (CBHA) manipulator is discussed by using the assumption of constant curvature [7], the singularity is also being handled during this approach. For solving the inverse kinematics of

\footnotetext{
${ }^{1}$ Authors are with Polytech Lille, CRIStAL, CNRS-UMR 9189, Avenue Paul Langevin, 59655 Villeneuve d'Ascq, France inderjeet.singheed.univ-lillel.fr, othman. lakhalduniv-lille1.fr, vincent.coelendpolytech-lille.net, rochdi.merzoukiapolytech-lille.fr

${ }^{2}$ Ecole Militaire Polytechnique, BP 17, Bordj EL Bahri, Algiers 16111, Algeria amara.yacine@gmail.com

Department of Mechanical and Industrial Engineering, Indian Institute of Technology Roorkee, Roorkee 247667, India pushpfmeditr.ac.in
}

continuum robots, a geometrical approach is represented and applied to OctArm in [8]. In this work, a procedure to find out the appropriate end points of sections is provided. For hyper-redundant manipulator kinematics, a modal-approach is developed by [9]. In this work, the hyper-redundant manipulator is represented by a backbone curve and to generate this backbone, an optimal curve is used. Optimization criteria is used to satisfy the task oriented constraints.

Some work has also been done to study qualitative models. The approximation of inverse kinematic model of a soft manipulator is done using a feed-forward Neural Networks (NN) [10]. Neural Network [11] based approach approximates the inverse kinematic model of CBHA robot. Hybrid approach, both quantitative as well as qualitative, is used in [12] for IKM of CBHA. In this work, an approximation of inverse kinematic equations of CBHA manipulator is done using a multilayer NN.

Obtaining a quantitative inverse kinematic model, for multisection continuum arm, from the forward kinematic equations is computationally infeasible because of high order of polynomials and high redundancy. Therefore, iterative approaches [13], [14] are preferable to find the solution of these type of problems.

In this paper, the main contribution is to perform the quantitative inverse kinematic models of CBHA manipulator with the help of Newton Raphson Method and Damped Least Square Method, in order to evaluate their performances in terms of accuracy and time costs. These proposed IKMs are validated and compared with already existing IKM for CBHA, using experimental setup. The IKMs are developed with free load condition, and are computed at position level.

The paper is organized as follows: Section II describes the CBHA manipulator. Inverse kinematic methods are discussed theoretically in section III. Section IV explains the procedure of validation and comparison of inverse kinematic methods with the existing methods. In Section V results are presented and discussed. Furthermore, conclusions are listed in section VI.

\section{COMPACt Bionic HANDling Assistant MANIPULATOR}

CBHA manipulator consists of two soft sections made up of elastic material (polyamide), a wrist which can rotate and a compliant gripper as labeled in Fig. 1. The main feature of CBHA manipulator is its flexibility. Each section is made up of three tubes, which are actuated by electro- 
pneumatic actuators. Pressure change inside the tubes leads to change in their respective lengths, leading to new position and orientation of the gripper.

If there is equal pressure in all three tubes of a section, then that section will extend in a straight line. Extension is limited because of an inextensible cable placed at the backbone of the manipulator. To create bending, differential pressures can be applied in the tubes. The elongation of each tube is measured from a wire-potentiometer. The wire-potentiometer sensors provide voltage proportional to the elongations $l_{i j}$. Where $i=1, \ldots, 3$ corresponds to the number of tube and $j=1$, 2 corresponds to the number of section. CBHA manipulator

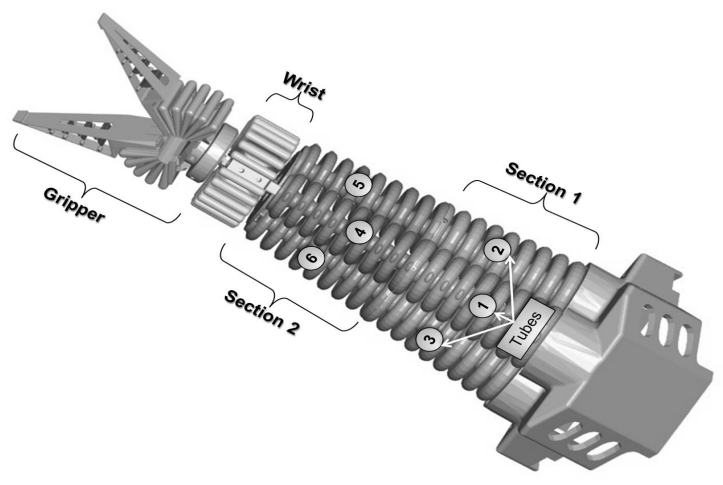

Fig. 1. CBHA manipulator description

is mounted on an omni-directional mobile base, known as Robotino. As mobile base as well as bionic manipulator, both are combined then it is known as RobotinoXT as shown in Fig. 2. It represents CBHA with the placement of frames, the first frame is attached at the base of the first section of CBHA, while the second frame is attached at the base of the second section, third at the end of the second section and fourth frame is at the tip point of gripper. All methods discussed in this paper considered modeling of CBHA from base of first section to the end of second section (frame 1 to frame 3).

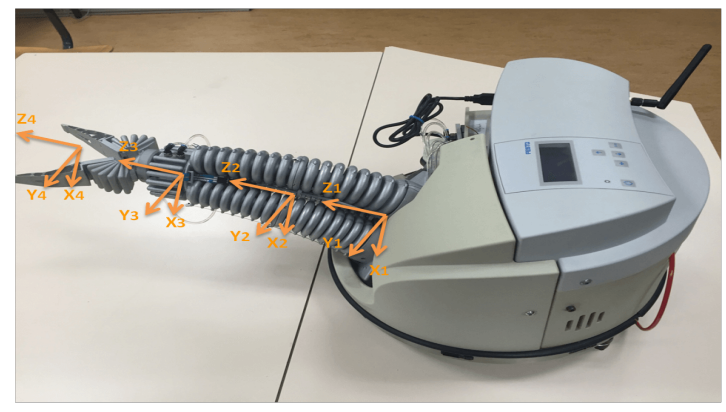

Fig. 2. Robotino XT with placement of frames on CBHA

\section{InVERse Kinematic Methods}

The inverse kinematic model of CBHA manipulator is developed with the help of two different quantitative methods. Both of these methods use forward kinematics model of CBHA [7]. This FKM is computed by considering the constant curvature of the backbone describing each section as shown in Fig. 3, the tip position $\sigma$ of manipulator is calculated as function of arc parameters as in (1).

$$
\sigma=f\left(L_{j}, \phi_{j}, r_{j}, \theta_{j}\right)
$$

Here, $L_{j}, r_{j}, \theta_{j}, \phi_{j}$ are backbone length, curvature radius, curvature angle and torsion angle of $j$ th section respectively. These arc parameters are calculated in terms of tube lengths $l_{i, j}$ as in (2).

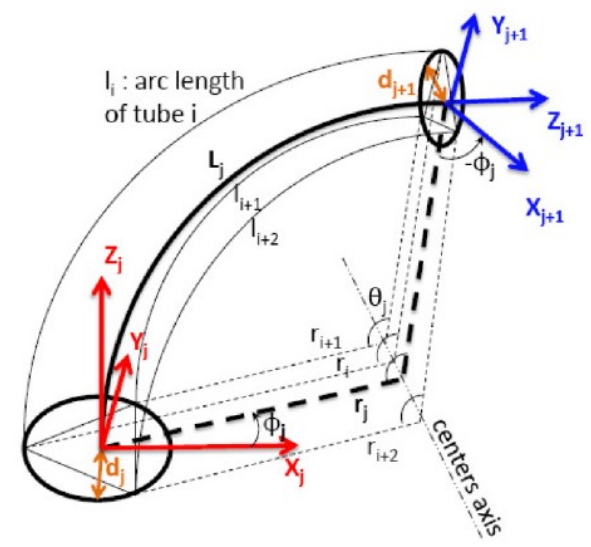

Fig. 3. Geometrical Representation of one Section of CBHA

$$
\left\{\begin{aligned}
L_{j} & =\frac{l_{i}+l_{i+1}+l_{i+2}}{3} \\
\phi_{j} & =\tan ^{-1}\left(\frac{\sqrt{3}\left(l_{i+2}-l_{i+1}\right)}{2 l_{i}-l_{i+1}-l_{i+2}}\right) \\
r_{j} & =\frac{\left(l_{i}+l_{i+1}+l_{i+2}\right)}{D_{j}} d_{j} \\
\theta_{j} & =\frac{D_{j}}{3 d_{j}} \\
D_{j} & =2 \sqrt{{l_{i}}^{2}+l_{i+1}{ }^{2}+l_{i+2}{ }^{2}-l_{i} l_{i+1}-l_{i} l_{i+2}-l_{i+1} l_{i+2}}
\end{aligned}\right.
$$

Final position $\sigma$ of manipulator is calculated by using transformations. Transformation matrix for one section of CBHA is given by (3).

$$
{ }_{j}^{j-1} T=\left[\begin{array}{cc}
R & P \\
0 & 1
\end{array}\right]
$$

Here,

$$
P=\left[\begin{array}{c}
r_{j} C \varphi_{j}\left(1-C \theta_{j}\right) \\
r_{j} S \varphi_{j}\left(1-C \theta_{j}\right) \\
r_{j} S \theta_{j}
\end{array}\right]
$$

and,

$$
R=\left[\begin{array}{ccc}
C^{2} \varphi_{j} C \theta_{j}+S^{2} \varphi_{j} & C \varphi_{j} S \varphi_{j}\left(C \theta_{j}-1\right) & C \varphi_{j} S \theta_{j} \\
C \varphi_{j} S \varphi_{j}\left(C \theta_{j}-1\right) & S^{2} \varphi_{j} C \theta_{j}+C^{2} \varphi_{j} & S \varphi_{j} S \theta_{j} \\
C \varphi_{j} S \theta_{j} & -S \varphi_{j} S \theta_{j} & C \theta_{j}
\end{array}\right]
$$

Where $C$ and $S$ are cos and sin respectively also $j$ is the section number, and $i$ is the tube number.

Therefore, transformation matrix for both sections of CBHA manipulator from frame 1 to frame 3 is:

$$
{ }_{3}^{1} T={ }_{2}^{1} T_{3}^{2} T
$$


Furthermore, the quantitative IKMs using FKM, are discussed.

\section{A. Newton Raphson Method}

Newton Raphson Method is an iterative method to find the better approximation to roots of a function. The functions used for calculation of inverse kinematic solution of CBHA, are taken from the forward kinematics of CBHA, singularity is already handled while computing forward kinematics [7]. Equations (5) indicate the $\mathrm{x}, \mathrm{y}$ and $\mathrm{z}$ coordinates of the end point of second section of CBHA, when viewed from the base coordinate frame. These equations are derived from Forward Kinematic Model of CBHA manipulator using transformations from workspace to tube space, through the configuration space using (4).

$$
\left\{\begin{aligned}
x= & r_{1}\left(-C \theta_{1}+1\right) C \phi_{1}+r_{2}\left(\left(S \phi_{1}\right)^{2}+\left(C \phi_{1}\right)^{2} C \theta_{1}\right) \\
& \left(-C \theta_{2}+1\right) C \phi_{2}+r_{2}\left(C \theta_{1}-1\right)\left(-C \theta_{2}+1\right) \\
& S \phi_{1} S \phi_{2} C \phi_{1}+r_{2} S \theta_{1} S \theta_{2} C \phi_{1} \\
y= & r_{1}\left(-C \theta_{1}+1\right) S \phi_{1}+r_{2}\left(\left(S \phi_{1}\right)^{2} C \theta_{1}+\left(C \phi_{1}\right)^{2}\right) \\
& \left(-C \theta_{2}+1\right) S \phi_{2}+r_{2}\left(C \theta_{1}-1\right)\left(-C \theta_{2}+1\right) \\
& S \phi_{1} C \phi_{1} C \phi_{2}+r_{2} S \phi_{1} S \theta_{1} S \theta_{2} \\
z= & r_{1} S \theta_{1}-r_{2}\left(-C \theta_{2}+1\right) S \phi_{1} S \theta_{1} S \phi_{2}-r_{2} \\
& \left(-C \theta_{2}+1\right) S \theta_{1} C \phi_{1} C \phi_{2}+r_{2} S \theta_{2} C \theta_{1}
\end{aligned}\right.
$$

Here $C$ and $S$ represent cos and sin respectively.

The functions used for Newton Raphson Method are as in (6), where $x, y$ and $z$ are used from Forward Kinematics of CBHA by using eq. (2) and (5) in term of lengths. $x_{t}, y_{t}$ and $z_{t}$ are the coordinates of point from the trajectory, recorded by stereo vision system, that has to be followed by robot. Therefore, these functions compute error.

$$
\left\{\begin{array}{l}
f=x_{t}-x \\
g=y_{t}-y \\
h=z_{t}-z
\end{array}\right.
$$

Let $E=[f ; g ; h]$ and $L=\left[l_{1} ; l_{2} ; l_{3} ; l_{4} ; l_{5} ; l_{6}\right]$ are column vectors. The Jacobian is computed as derivative of error vector $E$ with respect to the state space vector $L$ as:

$$
J=\frac{\partial(E)}{\partial(L)}
$$

As this is a mathematical model, and in mathematics the differentiation of one vector with respect to another vector is represented by term jacobian. Here, it is differentiation of error vector with respect to state space vector, thats why term jacobian is used. Therefore, it should not be confused with the traditional term jacobian used in robotics field. As the jacobian is not a square matrix and hence not invertible, a pseudo inverse $J^{\dagger}$ of jacobian matrix is calculated as:

$$
J^{\dagger}=J^{T}\left(J J^{T}\right)^{-1}
$$

Then the matrix form of Newton Raphson Algorithm is used to calculate the desired lengths from the given trajectory by using (9).

$$
l_{\text {new }}=l_{\text {old }}-J^{\dagger}[f ; g ; h]
$$

As, Newton Raphson method is iterative method, a tolerance need to define for the solution of this method. As tolerance will increase, the number of iterations will increase to reach that tolerance. In (9), $l_{\text {new }}$ are the lengths it calculates in the iteration and $l_{\text {old }}$ is the solution of lengths obtained during previous iteration. Therefore, this method continues to do iterations till the required tolerance of the solution is reached. Newton Raphson Method requires an initial approximation of the solution for its first iteration and the accuracy is more if the initial approximation is in the neighbourhood of the solution, in this case the initial approximation for the current solution is provided as solution of previous case (lengths correspond to the previous tip point).

For optimization, 'fmincon' function of MATLAB is used, as this function uses newton raphson method. Because of iterative nature of this method, optimization is used just to confirm that solution of all six lengths should be in their range. Minimum and maximum values of all lengths are provided as constraints and the cost function used is:

$$
Z=w_{1} f+w_{2} g+w_{3} h
$$

Basic penalty method of optimization is used, high weights are given and minimization of cost function is done for getting the solution of lengths within limits.

\section{B. Damped Least Square Method}

Damped Least square method is also a quantitative method, used to compute IKM using forward kinematic equations. This method is not an iterative method, it gives solution just using a mathematical expression. This method is used as in (11) in matrix form:

$$
\Delta l=J^{\dagger} \Delta e
$$

Here,

$\Delta \mathrm{e}=$ Target point $(x y z)-$ Current point $(x y z)$

$\Delta \mathrm{l}=$ Target lengths $\left(l_{1}, \ldots, l_{6}\right)-$ Current lengths $\left(l_{1}, \ldots, l_{6}\right)$

Each time, for computing solution (target lengths) for a trajectory point, current lengths are provided from the solution of lengths from previous trajectory point and the corresponding point as current point.

$$
J^{\dagger}=J^{T}\left(J J^{T}+\lambda^{2} I\right)^{-1}
$$

Here $\lambda$ represents the damping coefficient, the solution of (11) depends on the value of $\lambda$. The damping constant depends on the details of the multibody and the target positions and must be chosen carefully to make (11) numerically stable. Therefore, its value effects accuracy of results. Jacobian $J$ is calculated as:

$$
J=\frac{\partial(P)}{\partial(L)}
$$

Here $P=[x ; y ; z]$ is position vector. Therefore same like previous method, due to derivative of position vector with respect to state space vector, mathematical jacobian term is used as $J$. Equations (2) and (5) are utilized to take $x, y$ and $z$ in terms of lengths, for jacobian calculation.

Therefore, this method directly calculates the solution using (11). 


\section{EXPERIMENTAL VALIDATION}

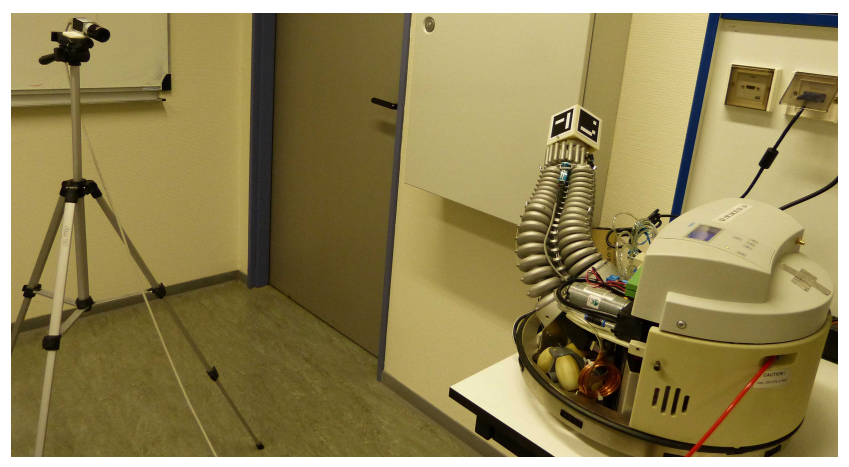

Fig. 4. Test bench

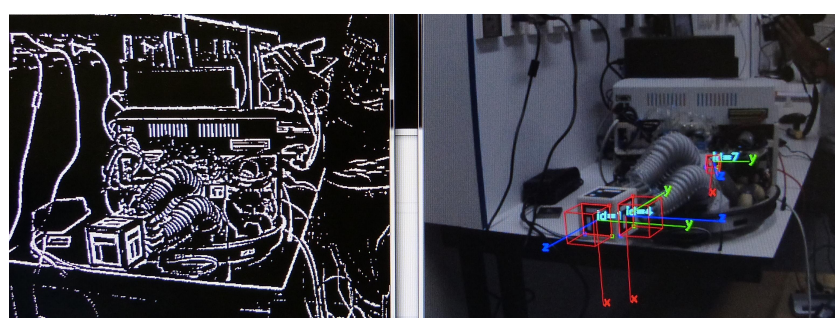

Fig. 5. Tip Point Tracking

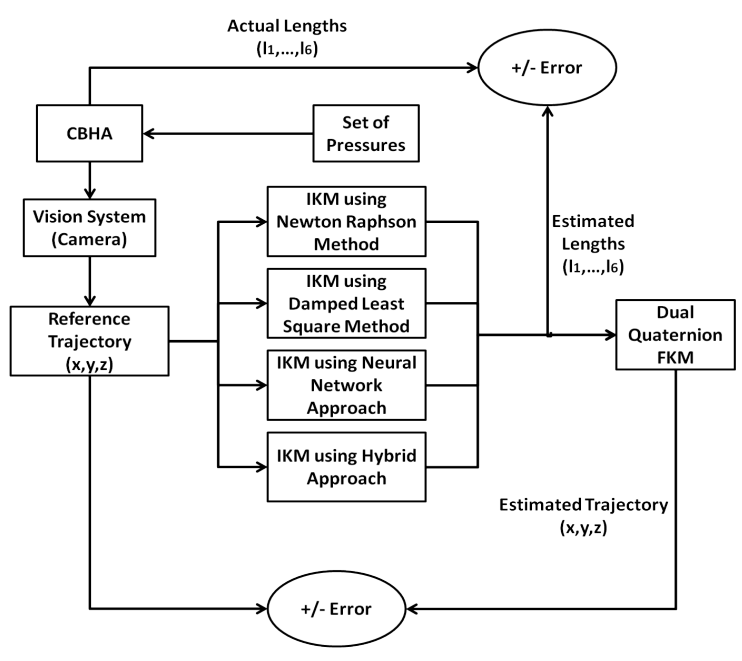

Fig. 6. Comparison of Inverse Kinematic Methods

Validation of proposed methods, requires a reference trajectory. A set of random pressures is applied to CBHA manipulator, then a trajectory with 100 points is recorded with the help of stereo vision system. For stereo vision, configuration of camera is: Basler acA645-100gc with the sony ICX414 CCD sensor. It delivers 100 frames per second at $(658 \mathrm{px} \times 492 \mathrm{px})$ resolution. Aruco markers are used for detection of tip point of CBHA manipulator as shown in Figs. 4 and 5. Neural Network based qualitative approach [11] is used to calculate IKM of CBHA. In this approach a
Distal Supervised Learning (DSL) technique is used to build a relation between effect and cause. Hybrid Approach used quantitative (Geometric) as well as qualitative (Neural Network) approaches to solve the inverse kinematics of CBHA [12]. In this approach, CBHA is considered, consisting of 17 vertebras, therefore each inter-vertebra is modeled as a parallel robot of type 3UPS-1UP (Universal-PrismaticSpherical). Fig. 6 shows the schematics of comparison of developed IKMs with the existing methods. The reference trajectory is provided to all IKMs and then the output lengths from the IKMs are transferred as an input to a Forward Kinematic Method (FKM) in order to compare between reference input trajectory and reconstructed trajectory from the modeling step (output of the FKM). Dual Quaternion Forward Kinematic Method [15] is used as Forward Kinematic Model, to be implemented with all the four methods of IKM.

\section{RESUlTS AND Discussions}

\section{A. Comparison of Lengths}

In case of CBHA manipulator, the main aim of inverse kinematic model is to calculate lengths $\left(l_{1}, \ldots ., l_{6}\right)$ by using reference trajectory as an input. The lengths are obtained from four different inverse kinematic methods. Fig. 7 compares the lengths $l_{1}, l_{2}$ and $l_{3}$ of first section of CBHA with reference length. Likewise, Fig. 8 compares the lengths $l_{4}$, $l_{5}$ and $l_{6}$ of second section of CBHA.

\section{B. Comparison of Euclidean Errors and Time Cost}

As in case of CBHA, same target position can be reached with more than one combination of lengths, so it is necessary to validate inverse kinematic methods using forward kinematic model. Using FKM, these four IKM are validated and compared on the basis of accuracy. Fig. 9 shows the trajectories from different methods after validating them using FKM, as well as the reference trajectory. From the different trajectories, the euclidean errors are computed with respect to reference. Fig. 10 shows errors in $x, y$ and $z$ axis for different methods. Time cost for each method is mentioned in table I.

TABLE I

MAXIMUM EUCLIDEAN ERRORS AND TIME COST PER POINT OF TRAJECTORY

\begin{tabular}{ccccc} 
Method & $\begin{array}{c}X \text {-error } \\
(\mathrm{mm})\end{array}$ & $\begin{array}{c}Y \text {-error } \\
(\mathrm{mm})\end{array}$ & $\begin{array}{c}Z \text {-error } \\
(\mathrm{mm})\end{array}$ & $\begin{array}{c}\text { Time Cost } \\
(\mathrm{sec})\end{array}$ \\
\hline Newton Raphson & 0.146 & 0.219 & 0.975 & 0.2335 \\
Damped Least Square & 16.208 & 18.104 & 24.155 & 0.0005 \\
Neural Network & 11.791 & 3.491 & 9.547 & 0.0138 \\
Hybrid & 15.519 & 5.392 & 9.930 & 0.0120 \\
\hline
\end{tabular}

\section{Discussions}

As in case of CBHA, there exists infinite number of solutions of IKM. From the comparison of lengths, it seems that lengths $\left(l_{1}, \ldots ., l_{6}\right)$ from all methods follow approximately the same variation, as the reference lengths.

In terms of accuracy, Newton Raphson method is the more 


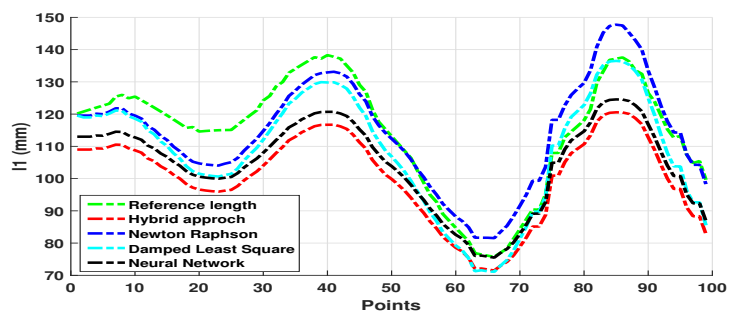

(a) Comparison of length $l_{1}$

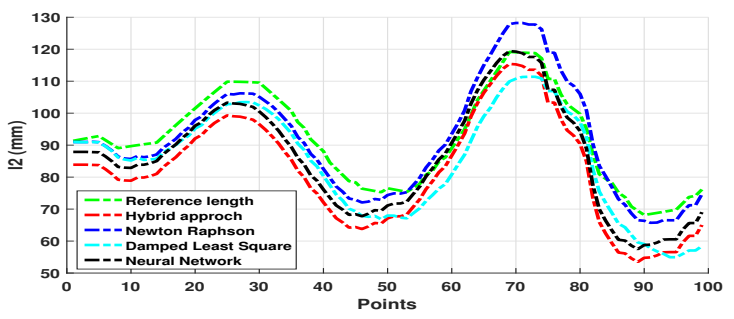

(b) Comparison of length $l_{2}$

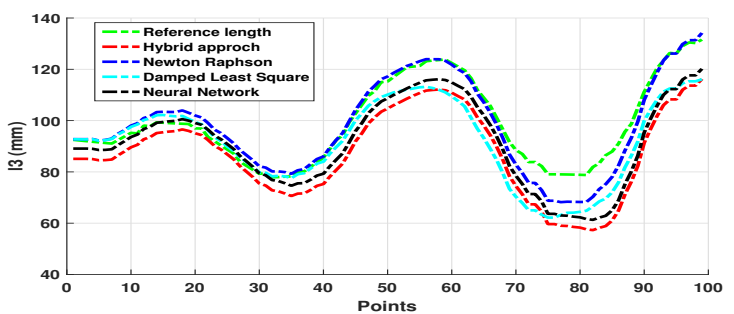

(c) Comparison of length $l_{3}$

Fig. 7. Comparison of lengths $l_{1}, l_{2}$ and $l_{3}$ of section 1 of CBHA

accurate, followed by Neural Network approach, Hybrid Method and Damped Least Square Method. Newton Raphson and Damped Least Square, both are quantitative methods but the former is more accurate. In Newton Raphson, the solution depends on the initial approximation of solution provided but in Damped Least Square, solution depends on initial approximation as well as value of damping coefficient $\lambda$. A global value 0.5 is taken for $\lambda$, for the best fit of overall trajectory. But if an algorithm can be developed to calculate the different values of $\lambda$ for different inputs, then the accuracy of Damped Least Square can be increased. Damped Least Square approach gives accurate results in starting region but after, the errors are diverging, therefore for the region having more errors, different value of $\lambda$ can reduce the errors, but this is not considered in this work. Neural Network Approach is accurate than Hybrid Approach, both of these approaches use NN, Neural Network Approach is totally based on NNs but Hybrid Method developed inverse kinematic equations of parallel serial robot analytically, it uses NN only to approximate these equations because of high non-linearities of equations, this approximation makes this approach some less accurate than Neural Network approach. Time Cost for Damped Least Square method is the least but for Newton Raphson Method, it is more. This time cost is for calculation of one target point. Among both quantitative methods, Newton Raphson is an iterative method, so it does

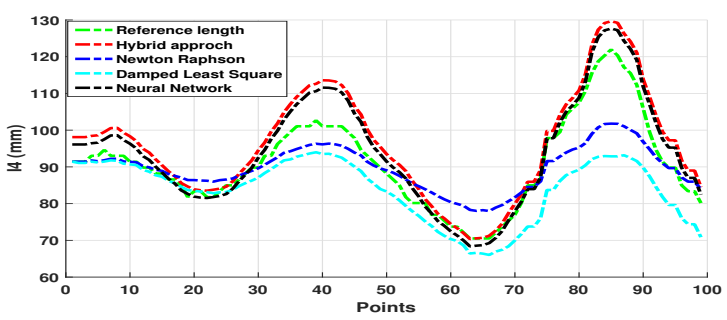

(a) Comparison of length $l_{4}$

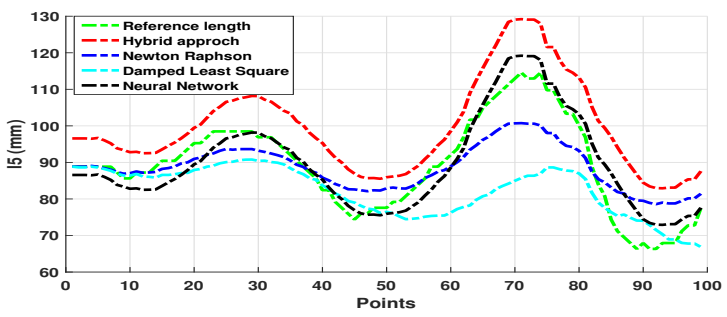

(b) Comparison of length $l_{5}$

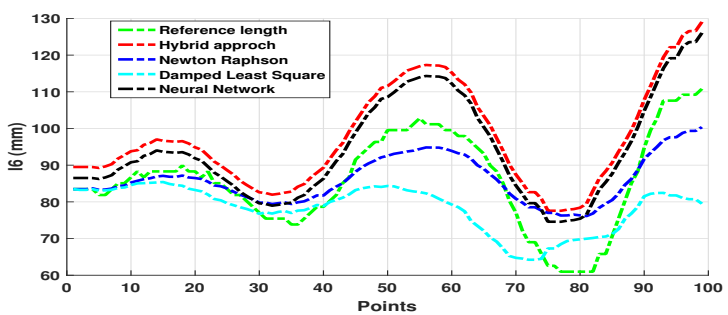

(c) Comparison of length $l_{6}$

Fig. 8. Comparison of lengths $l_{4}, l_{5}$ and $l_{6}$ of section 2 of CBHA

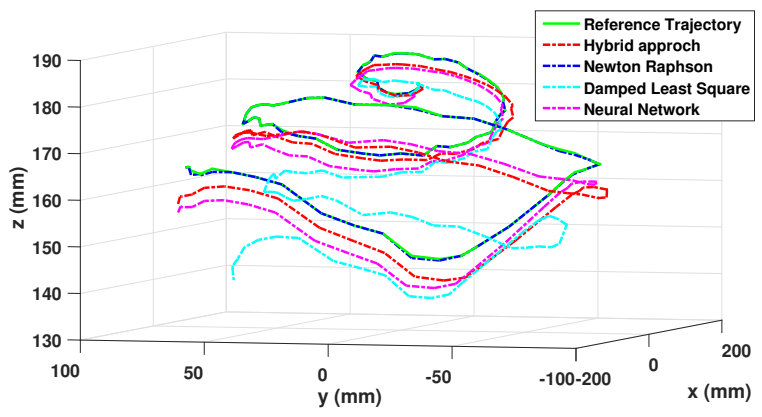

Fig. 9. Trajectory comparison with reference

iterations for each output, to reach an accurate solution but Damped Least Square Method uses a mathematical relation for calculations. In this case, Newton Raphson Method takes maximum three iterations to converge to solution for a tolerance of $10^{-3} \mathrm{~mm}$. Therefore, Newton Raphson Method is taking more time. Among qualitative methods, both methods take approximately same time for inverse kinematic solution but to develop these methods, the learning approach is used, which also takes time.

In case of Neural Network Approach, The database of NN is obtained with a pause of $8 \mathrm{sec}$ between two movements. So, to generate the database, CBHA must function for 8 hours, this can deteriorate the robot. If there are three sections or 


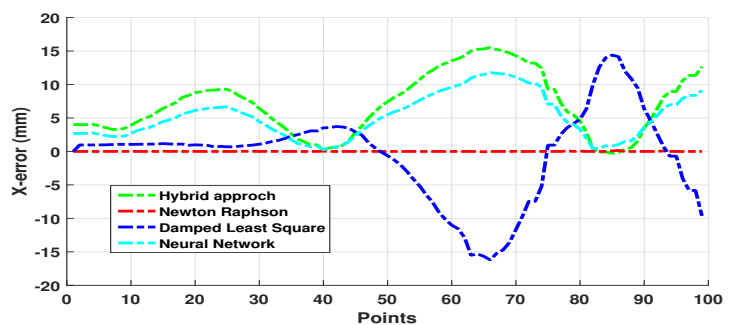

(a) Euclidean Error in $\mathrm{x}$ axis

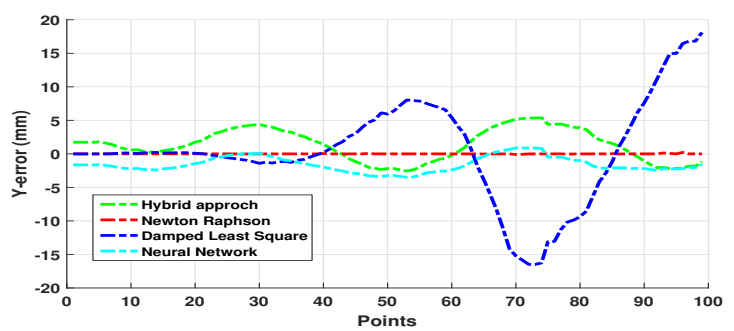

(b) Euclidean Error in y axis

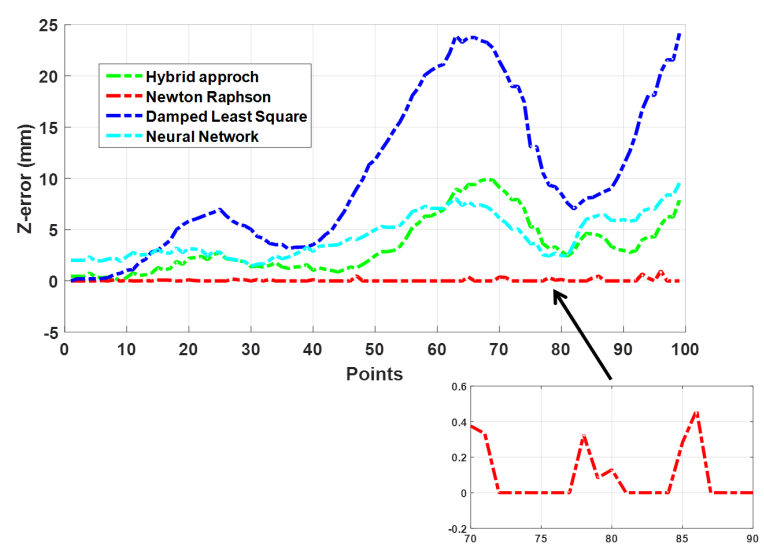

(c) Euclidean Error in $\mathrm{z}$ axis

Fig. 10. Euclidean errors

more, the learning base is of $9^{6}$ samples, which is huge data and takes a lot of time. In both qualitative methods, The learning base is divided randomly: $70 \%$ for the training set, $15 \%$ for the validation set, and $15 \%$ for the test set. The training and validation sets are used during the learning phase and the test set is only employed to assess the performance of the neural network model.

Hence the above discussion implies, the high accuracy of Newton Raphson Method can compromise with time cost because in most of applications accuracy is an important factor. Therefore, Newton Raphson Approach is recommended to use for an accurate IKM of CBHA manipulator.

\section{CONCLUSION}

In this paper, two different methods to compute IKM for CBHA, are discussed, validated and compared in terms of accuracy and time cost with existing IKM for CBHA. Advantages and disadvantages of each method are discussed to choose an appropriate method to develop the kinematic control of CBHA in closed loop. This work is done for
CBHA, consisting of two sections but it is important that in future we evaluate the performance of these methods for more than two sections and also to develop the frame work of the cooperation of multiple CBHAs.

\section{ACKNOWLEDGMENT}

This work was supported by the joint Collaborative Research Project between CNRS in France and DST in India (PRC CNRS-DST 2016-2018). The authors want to thank Dr. Reinhard Pittschellis and Dr. Dirk Pensky from Festo Didactic company in Denkendorf (Germany) for their technical assistance and support on RobotinoXT.

\section{REFERENCES}

[1] M. Sfakiotakis, A. Kazakidi, N. Pateromichelakis, and D. P. Tsakiris, "Octopus-inspired eight-arm robotic swimming by sculling movements," in Robotics and Automation (ICRA), 2013 IEEE International Conference on. IEEE, 2013, pp. 5155-5161.

[2] R. Behrens, M. Poggendorf, E. Schulenburg, and N. Elkmann, "An elephant's trunk-inspired robotic arm-trajectory determination and control," in Robotics; Proceedings of ROBOTIK 2012; 7th German Conference on. VDE, 2012, pp. 1-5.

[3] I. D. Walker, D. M. Dawson, T. Flash, F. W. Grasso, R. T. Hanlon, B. Hochner, W. M. Kier, C. C. Pagano, C. D. Rahn, and Q. M. Zhang, "Continuum robot arms inspired by cephalopods," in Proc. SPIE, vol. 5804, 2005, pp. 303-314.

[4] I. S. Godage, E. Guglielmino, D. T. Branson, G. A. MedranoCerda, and D. G. Caldwell, "Novel modal approach for kinematics of multisection continuum arms," in Intelligent Robots and Systems (IROS), 2011 IEEE/RSJ International Conference on. IEEE, 2011, pp. 1093-1098.

[5] M. Hannan and I. Walker, "Novel kinematics for continuum robots," in 7th International Symposium on Advances in Robot Kinematics, 2000, pp. 227-238.

[6] Q. Zhao and F. Gao, "Design and analysis of a kind of biomimetic continuum robot," in Robotics and Biomimetics (ROBIO), 2010 IEEE International Conference on. IEEE, 2010, pp. 1316-1320.

[7] C. Escande, R. Merzouki, P. M. Pathak, and V. Coelen, "Geometric modelling of multisection bionic manipulator: Experimental validation on robotinoxt," in Robotics and Biomimetics (ROBIO), 2012 IEEE International Conference on. IEEE, 2012, pp. 2006-2011.

[8] S. Neppalli, M. A. Csencsits, B. A. Jones, and I. Walker, "A geometrical approach to inverse kinematics for continuum manipulators," in Intelligent Robots and Systems, 2008. IROS 2008. IEEE/RSJ International Conference on. IEEE, 2008, pp. 3565-3570.

[9] G. S. Chirikjian and J. W. Burdick, "A modal approach to hyperredundant manipulator kinematics," IEEE Transactions on Robotics and Automation, vol. 10, no. 3, pp. 343-354, 1994.

[10] M. Giorelli, F. Renda, G. Ferri, and C. Laschi, "A feed-forward neural network learning the inverse kinetics of a soft cable-driven manipulator moving in three-dimensional space," in Intelligent Robots and Systems (IROS), 2013 IEEE/RSJ International Conference on. IEEE, 2013, pp. 5033-5039.

[11] A. Melingui, O. Lakhal, B. Daachi, J. B. Mbede, and R. Merzouki, "Adaptive neural network control of a compact bionic handling arm," IEEE/ASME Transactions on Mechatronics, vol. 20, no. 6, pp. 2862$2875,2015$.

[12] O. Lakhal, A. Melingui, and R. Merzouki, "Hybrid approach for modeling and solving of kinematics of a compact bionic handling assistant manipulator," IEEE/ASME Transactions on Mechatronics, vol. 21, no. 3, pp. 1326-1335, 2016.

[13] J. M. Hollerbach and C. W. Wampler, "The calibration index and taxonomy for robot kinematic calibration methods," The international journal of robotics research, vol. 15, no. 6, pp. 573-591, 1996.

[14] A. Goldenberg, B. Benhabib, and R. Fenton, "A complete generalized solution to the inverse kinematics of robots," IEEE Journal on Robotics and Automation, vol. 1, no. 1, pp. 14-20, 1985.

[15] I. Singh, O. Lakhal, and R. Merzouki, "Towards extending forward kinematic models on hyper-redundant manipulator to cooperative bionic arms," in Journal of Physics: Conference Series, vol. 783, no. 1. IOP Publishing, 2017, p. 012056. 